\title{
The ATLAS Level-1 Central Trigger Processor
}

\author{
P. Borrego Amaral*, N. Ellis*, P. Farthouat*, P. Gällnö*, J. Haller*, A. Krasznahorkay*†, \\ T. Maeno*, T. Pauly*, H. Pessoa Lima Junior ${ }^{\ddagger \S}$, I. Resurreccion Arcas*, G. Schuler*, \\ J. M. de Seixas ${ }^{\ddagger}$, R. Spiwoks*, R. Torga Teixeira*, T. Wengler* \\ *CERN, Geneva, Switzerland \\ ${ }^{\dagger}$ University of Debrecen, Hungary

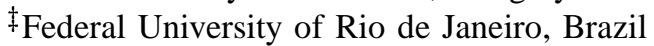 \\ $\S$ Brazilian Center for Physics Research, Brazil
}

\begin{abstract}
ATLAS is a multi-purpose particle physics detector at CERN's Large Hadron Collider where two pulsed beams of protons are brought to collision at very high energy. There are collisions every $25 \mathrm{~ns}$, corresponding to a rate of $40 \mathrm{MHz}$. A three-level trigger system reduces this rate to about $200 \mathrm{~Hz}$ while keeping bunch crossings which potentially contain interesting processes. The Level-1 trigger, implemented in electronics and firmware, makes an initial selection in under $2.5 \mu$ s with an output rate of less than $100 \mathrm{kHz}$. A key element of this is the Central Trigger Processor (CTP) which combines trigger information from the calorimeter and muon trigger processors to make the final Level-1 accept decision in under $100 \mathrm{~ns}$ on the basis of lists of selection criteria, implemented as a trigger menu. Timing and trigger signals are fanned out to all sub-detectors, while busy signals from all sub-detector read-out systems are collected and fed into the CTP in order to throttle the generation of Level-1 triggers.
\end{abstract}

\section{The ATLAS LEVEL-1 TRIgGer System}

The ATLAS Level-1 trigger [1] is a synchronous system operating at the bunch crossing (BC) frequency $(40.08 \mathrm{MHz})$ of CERN's Large Hadron Collider. It uses information on clusters and global energy in the calorimeters and from tracks in dedicated muon trigger detectors. An overview of the ATLAS Level-1 trigger is shown in Fig. 1.

The calorimeter [2] and muon [3][4] trigger processors provide trigger information to the Central Trigger Processor (CTP). The CTP forms the Level-1 Accept decision (L1A) and fans it out to Timing, Trigger and Control (TTC) partitions. In the ATLAS experiment there are about 40 TTC partitions. Each partition contains one Local Trigger Processor (LTP) [5], a TTC system [6], and a busy tree which allows to throttle the generation of L1As and which is based on the ROD_BUSY module [7]. The CTP provides trigger summary information to the Level-2 trigger system and to the data acquisition system [8]. It is configured, controlled and monitored by the Online System [9][10].

\section{THE FUNCTION OF THE CTP}

The CTP receives trigger information from the calorimeter and muon trigger processors, which consists of multiplicities for electrons/photons, taus/hadrons, jets, and muons, and of flags for total transverse energy, total missing transverse energy, and total jet transverse energy. Additional inputs are provided for special triggers such as a filled-bunch trigger

\begin{tabular}{lccr}
\hline \hline Trigger Item & Mask & Priority & Pre-scaling \\
\hline 1MU6 & On & Low & 1000 \\
2MU6 & On & High & 1 \\
1EM10 AND XE20 & On & Low & 1 \\
\hline \hline
\end{tabular}

TABLE I

AN EXAMPLE OF PART OF A LEVEL-1 TRIGGER MENU: MU STANDS FOR MULTIPLICITY TRIGGER INPUT FOR MUONS, EM FOR MULTIPLICITY TRIGGER INPUT FOR ELECTRONS/PHOTONS, AND XE FOR TRIGGER INPUT FOR MISSING TRANSVERSE ENERGY.

based on beam pick-up monitors, and a minimum-bias trigger based on scintillator counters.

All triggers and their thresholds are programmable. Several thresholds are used concurrently for each type of trigger information. Up to 160 trigger inputs can be taken into account by the CTP at any one time. The total number of trigger inputs can be higher because a programmable selection can be made at the input to the CTP.

The CTP generates L1As derived from the trigger inputs according to the Level-1 trigger menu. The menu consists of up to 256 trigger items each of which is a combination of one or more conditions on the trigger inputs and the internal triggers provided by the CTP (two random triggers, two prescaled clocks, and eight triggers for programmable groups of bunch crossings). If, e.g, EM10 symbolises the multiplicity trigger input for electrons/photons with a transverse energy of at least $10 \mathrm{GeV}$, then 1EM10 symbolises the condition of there being at least one electron/photon above that threshold. 256 different conditions can be defined, and several conditions can be combined to form a trigger item. Each trigger item further has a mask, a priority (for the dead-time generated by the CTP), and a pre-scaling factor. The L1A is the logical OR of all trigger items. An example of part of a Level-1 trigger menu is shown in Tab. I.

The CTP provides an 8-bit trigger-type word with each L1A which indicates the type of trigger and can be used to select options in event data processing in the sub-detector front-end electronics.

The CTP specification aims for L1A generation within 100 ns (time between input to the CTP and output of the L1A signal), corresponding to 4 BCs. The menu used for the 


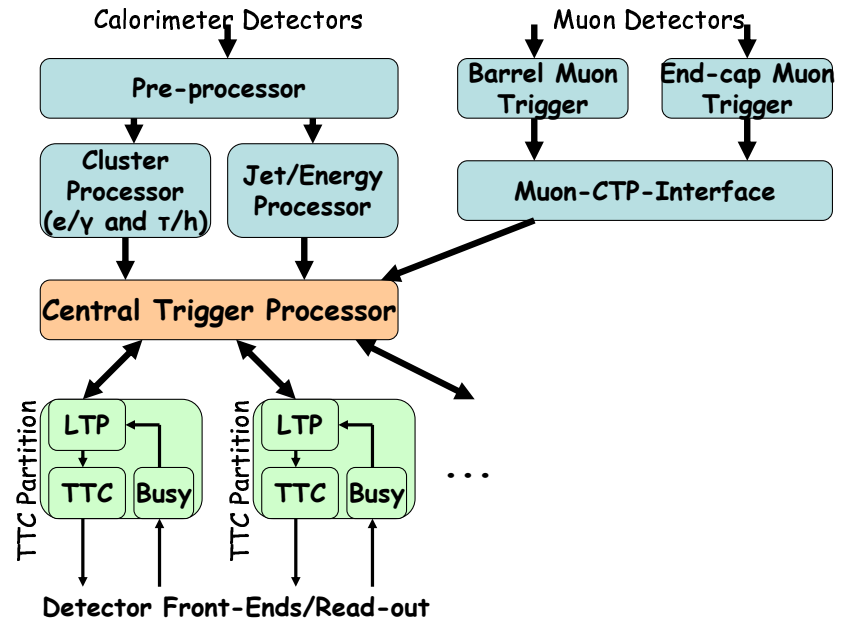

Fig. 1. Overview of the ATLAS Level-1 Trigger.

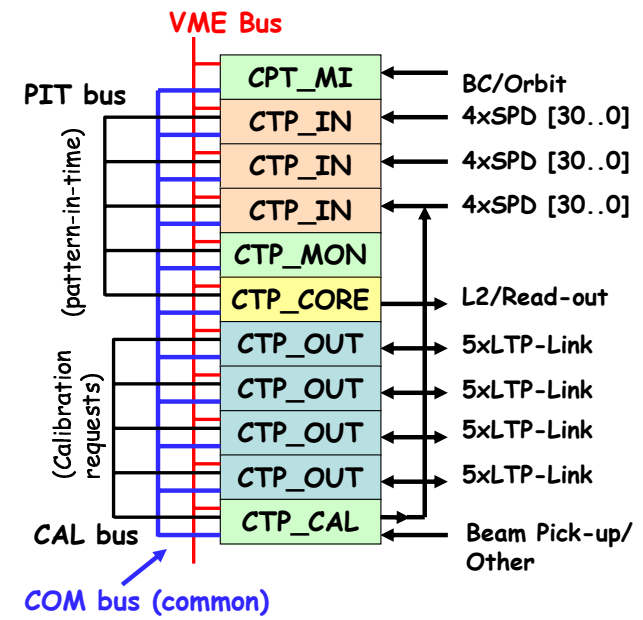

Fig. 2. The CTP design with its modules, backplanes, and signals. trigger formation is likely to change frequently depending on the physics, beam and detector conditions. High flexibility has to be provided for the L1A generation.

The CTP sends, at every L1A, information to the Regionof-Interest Builder (RoIB) in the Level-2 trigger for guidance of the Level-2 trigger algorithms. It also provides information to the Read-Out System of the data acquisition system. This information is a superset of the information to the RoIB and can contain data for several bunches before and after the triggering bunch for debugging and monitoring purposes.

The CTP provides monitoring data: snapshots of incoming data; bunch-by-bunch scalers of inputs; and scalers of trigger inputs and trigger items before and after pre-scaling integrated over all bunches.

The CTP is, like all other components of the Level-1 trigger, configured, controlled and monitored by the Online System [10].

\section{THE DESIGN OF THE CTP}

The CTP consists of several different modules which are housed in a single 9U VME64x crate. In addition to VMEbus, the CTP modules use custom buses for the synchronised and aligned trigger inputs (PITbus, PIT = pattern in time), for the common timing and trigger signals (COMbus), and for the subdetector calibration requests (CALbus). The different types of modules are described in some detail below. An overview of the design of the CTP is shown in Fig. 2.

The CTP machine interface module (CTP_MI) receives timing signals from the LHC accelerator via the TTCmi [6], or generates them locally. It controls and monitors the internal and external busy signals. The CTP_MI sends the timing signals to the COMbus.

The CTP input module (CTP_IN) receives trigger inputs from the trigger processors and other sources. It synchronises the trigger inputs with respect to the internal clock, checks their parity, and aligns them with respect to the bunch-crossing identifier [11]. The CTP_IN selects and routes trigger inputs to be sent to the PITbus. It can capture a history of the trigger inputs in a test memory or provide trigger inputs from a test memory. The CTP_IN also monitors the trigger inputs using scalers that integrate over all bunches.

The CTP core module (CTP_CORE) receives the 160 trigger inputs from the PITbus. It combines them with additional internal triggers. All trigger inputs are combined using several look-up tables to form up to 256 trigger conditions, such as two random triggers, two pre-scaled clocks, and eight triggers for programmable groups of bunch crossings. The trigger conditions are combined using content-addressable memories to form up to 256 trigger items. Those trigger items can be prescaled by individually programmable 24-bit down-counters, and gated with individually programmable masks, dead-time and busy signal. The dead-time is the combination of a simple dead-time algorithm and a programmable selection of either of two complex dead-times (high and low priority dead-times). The L1A is subsequently formed by a logic OR of all trigger items.

The CTP_CORE sends the trigger results to the COMbus. It also sends information to the RoIB of the Level-2 trigger system and to the read-out system of the data acquisition system.

The CTP monitoring module (CTP_MON) receives and synchronises the trigger inputs from the PITbus. It decodes and selects the trigger inputs to be monitored. The CTP_MON scales trigger information on a bunch-by-bunch basis.

The CTP output module (CTP_OUT) receives timing and trigger signals from the COMbus and fans them out to the subdetector LTPs. The CTP_OUT also receives busy signals and calibration requests from the LTPs. It masks and monitors the busy signals, and provides them to the COMbus. It provides the calibration requests to the CALbus.

The CTP calibration module (CTP_CAL) time-multiplexes the calibration requests on the CALbus and sends them to the CTP_IN. The sub-detectors are allocated LHC orbits during which they may issue calibration requests. The CTP_CAL also contains other external inputs for beam pick-up monitors and 


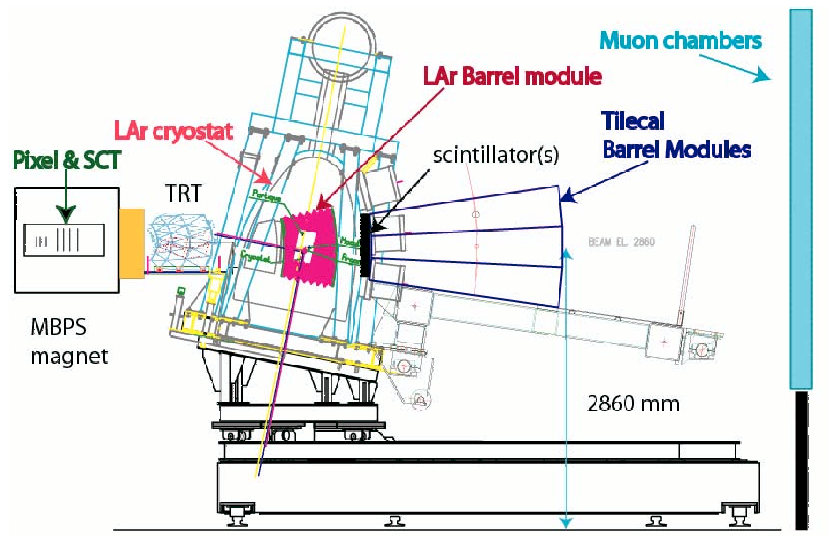

Fig. 3. The Setup of the ATLAS Combined Test-Beam.

test triggers which are sent via cable to the CTP_IN.

\section{The CTP IN The ATLAS Combined Test-BeAm}

During 2004 the ATLAS combined beam-test activity focused on testing prototypes and final modules of all subdetectors, including the full trigger and data acquisition chain. A sketch of the setup from a mechanical point of view is shown in Fig. 3.

During a period of $25 \mathrm{~ns}$ structured beam the calorimeters provided real data to the calorimeter trigger processors, which in turn provided trigger information via two Common Merger Modules (CMM) [2] to the CTP. Similarly, the muon trigger detectors, Resistive Plate Chambers (RPC) for the barrel [3] and Thin-Gap Chambers (TGC) for the end-cap [4], provided data to the Muon-CTP-Interface (MUCTPI) [12] which summarised the muon trigger information and sent it to the CTP. In addition, scintillator triggers from the beam instrumentation could also be used in the CTP.

The CTP was used with one CTP_MI, one CTP_IN (out of up to three), one CTP_CORE, one CTP_OUT (of up to four) and one CTP_MON. The CTP_OUT was connected to one LTP from which the L1A and other timing signals were fanned out to the sub-detector front-end electronics. An overview of the setup of the Level-1 trigger during the October 2004 run with $25 \mathrm{~ns}$ beam is shown in Fig. 4. A photograph of the CTP in the ATLAS combined test-beam is shown in Fig. 5.

In total, the CTP_IN received 46 bits of trigger information:

- $4 \times 3$ bits of e/ $\gamma$ and $4 \times 3$ bits of jet multiplicities;

- 1 bit total transverse energy;

- $6 \times 3$ bits of muon multiplicities;

- $3 \times 1$ bit of scintillator triggers.

The 46 trigger input bits were used to form 18 trigger items in the CTP_CORE. They could be pre-scaled and masked correctly. The L1A was used as trigger for the read-out of the combined sub-detectors. An inspection of the data taken by the sub-detectors showed that this worked correctly.

The scintillator triggers were used as time reference and the full latency of the trigger system could be measured for the TGC muon trigger. A projection to the final ATLAS Level-1

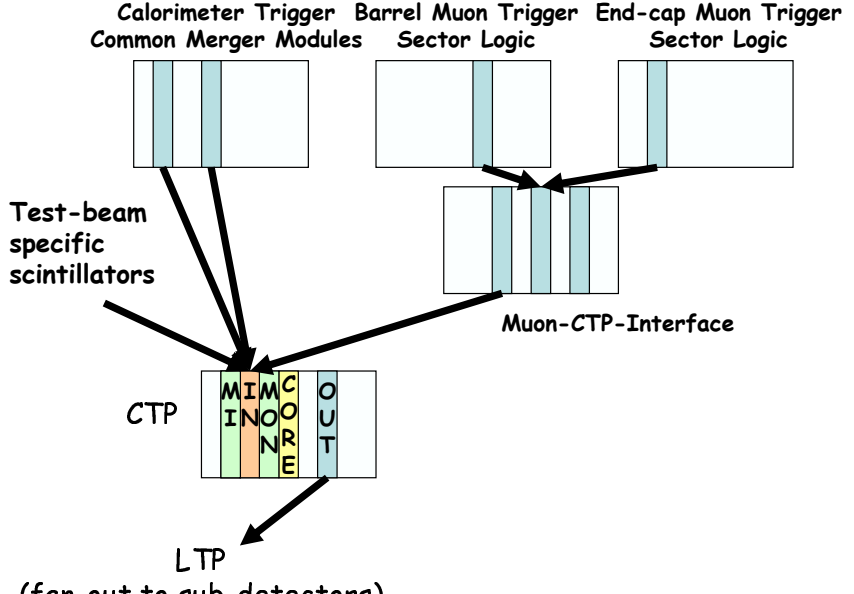

(fan-out to sub-detectors)

Fig. 4. The Level-1 Trigger at the ATLAS Combined Test-Beam.

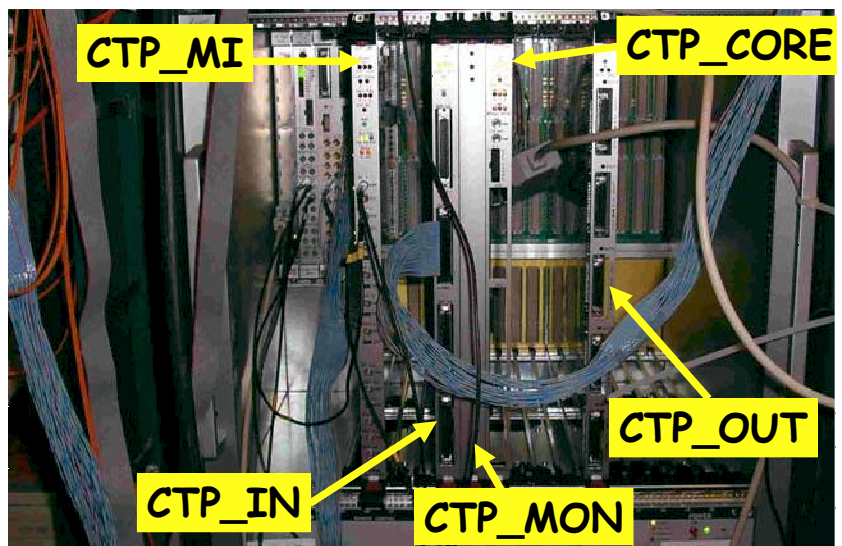

Fig. 5. The CTP at the ATLAS Combined Test-Beam.

latency (from the interaction point to the arrival of the L1A at the front-end of the TGC chambers), including cable length and time-of-flight corrections, gave a value of $2.13 \mu \mathrm{s}$, which is well within the budget of $2.5 \mu \mathrm{s}$.

In addition, the latency of the CTP was measured to be $125 \mathrm{~ns}$ (from the input of the CTP_IN to the output of the L1A at the CTP_OUT; cable delays have been taken into account), with non-optimised timing in the CTP. The measurement was repeated in the laboratory and after further optimisation a latency of $95 \mathrm{~ns}$ was measured.

\section{CONCLUSION}

The CTP has been tested during the ATLAS combined test-beam with triggers from detectors using a $25 \mathrm{~ns}$ bunch structure particle beam. The CTP_CORE was used during the test-beam to generate triggers for read-out of the combined sub-detectors based on 46 trigger input bits and 18 trigger items. The latency of the CTP was measured to be $95 \mathrm{~ns}$. Work on the CTP will continue in the laboratory concerning read-out, firmware, software and monitoring. The CTP will be available for commissioning of ATLAS in September 2005. 


\section{ACKNOWLEDGEMENTS}

We would like to thank our colleagues from the calorimeter trigger, barrel muon trigger, end-cap muon trigger, and data acquisition groups for their friendly and competent collaboration without which the work presented in this article would not have been possible.

\section{REFERENCES}

[1] The ATLAS Collaboration, "First-level Trigger Technical Design Report,” CERN/LHCC/98-14, June 1998.

[2] "The ATLAS Level-1 Calorimeter Trigger," http://hepwww.rl.ac.uk/Atlas-L1/Home.html.

[3] "The ATLAS Level-1 Barrel Muon Trigger" http: //sunset.romal.infn.it/muonl1/docs/publications, in particular the presentation "Slice Test Results of the ATLAS Barrel Muon Level-1 Trigger."

[4] "The ATLAS Level-1 End-cap Muon Trigger," http: / / at las .web.cern.ch/Atlas/project/TGC/www/doc/MuonEndcap_rev$01 \cdot \mathrm{pdf}$.
[5] P. Borrego Amaral et al., "The ATLAS Local Trigger Processor," CERN/ATL-DAQ-2004-016.

[6] "The TTC System," http://ttc.web.cern.ch/TTC/intro.html.

[7] P. G̈alln”o, “ROD Busy Module Technical Manual,” https: //edms.cern.ch/document/456631/4.

[8] "The ATLAS Dataflow System," http://at las.web. cern. ch/Atlas/GROUPS /DAQTRIG/dataflow. html.

[9] "The ATLAS Online System," http://atlas-onlsw.web.cern.ch/Atlas-onlsw/.

[10] R. Spiwoks, "Data Acquisition for the ATLAS Level-1 Central Trigger Processor," http://edms.cern.ch/document/312843.

[11] R. Spiwoks et al., "The ATLAS Level-1 Trigger Timing Setup," Poster presentation no. 1010, these proceedings.

[12] N. Ellis et al., “The ATLAS Level-1 Muon to Central Trigger Processor Interface (MUCTPI)," 8th Workshop on Electronics for LHC Experiments, Colmar, France, September 2002. 